AperTO - Archivio Istituzionale Open Access dell'Università di Torino

\title{
Exploratory study on the occurrence and dynamics of yeast-mediated nicotinamide riboside production in craft beers
}

This is a pre print version of the following article:

Original Citation:

Availability:

This version is available http://hdl.handle.net/2318/1788648

since 2021-05-10T14:50:56Z

Published version:

DOI:10.1016/j.Iwt.2021.111605

Terms of use:

Open Access

Anyone can freely access the full text of works made available as "Open Access". Works made available under a Creative Commons license can be used according to the terms and conditions of said license. Use of all other works requires consent of the right holder (author or publisher) if not exempted from copyright protection by the applicable law. 
1 Exploratory study on the occurrence and dynamics of yeast-mediated nicotinamide riboside

2 production in craft beers

3

4 Cristiana Garofalo ${ }^{1}$, Riccardo Sabbatini ${ }^{1}$, Federica Zamporlini ${ }^{1}$, Gabriele Minazzato ${ }^{1}$, Ilario $5 \quad$ Ferrocino $^{2}$, Lucia Aquilanti ${ }^{1}$, Nadia Raffaelli $^{1 *}$, Andrea Osimani ${ }^{1 *}$

6

7 Dipartimento di Scienze Agrarie, Alimentari ed Ambientali, Università Politecnica delle Marche, 8 via Brecce Bianche, 60131 Ancona, Italy

$9 \quad{ }^{2}$ DISAFA - Microbiology and food technology sector, University of Turin, Largo Paolo Braccini, 2 , 1010095 Grugliasco, Torino, Italy

11

12

13

14

15

16

17

18

19

$25 *$ Corresponding authors

26 E-mail addresses: a.osimani@ univpm.it (AO); n.raffaelli@ staff.univpm.it (NR) 
Several health benefits are related to the administration of nicotinamide riboside (NR), a form of Vitamin B3, and its precursors nicotinamide mononucleotide (NMN) and $\mathrm{NAD}^{+}$. Therefore, considerable interest is currently devoted to the potential therapeutic value of their supplementation, thus justifying scientific studies on the distribution of these molecules in foods and beverages. In this study, the three vitamers were quantitatively analyzed in ten craft beers for the first time. All beers from different commercial $S$. cerevisiae strains contained $\mathrm{NAD}^{+}$. NR, NMN and NAD ${ }^{+}$were mostly present in beers produced with Saccharomyces cerevisiae strain US-05. Interestingly, the three vitamers were not detectable in beers produced with a commercial strain of Saccharomyces pastorianus. Data from laboratory-scale beer production using S. cerevisiae strain US-05 showed that the addition of hops during the fermentation process significantly increased NR production. The rapid increase in NR formation only occurred if both hops and yeast were present, and the burst was also confirmed in fermentations trials performed with $S$. cerevisiae strain $\mathrm{CBS}_{1171^{\mathrm{T}}}$ and by replacing wort with YPD medium. The experimental model proposed in the present study can serve as baseline for further research aimed at investigating the yeast-hop interaction at metabolic and molecular levels. In addition to highlighting the potentialities of microorganisms to act as biological factories for beneficial molecules to humans, these findings open new intriguing perspectives for the development of innovative fermented foods naturally enriched in NR and its precursors. synergy 


\section{Introduction}

Vitamin B3, which is also called Niacin or vitamin PP (Pellagra-Preventing), includes nicotinic acid (NA), its amide nicotinamide (Nam), and nicotinamide riboside (NR), the last discovered form (Bieganowski \& Brenner, 2004). Once taken with the diet, NA, Nam and NR are transported inside the cells where they can be transformed into $\mathrm{NAD}^{+}$, which represents the biologically active form of vitamin $\mathrm{B} 3 . \mathrm{NAD}^{+}$itself is present in food together with the phosphorylated form of NR, i.e., nicotinamide mononucleotide $(\mathrm{NMN})$. In the human gut, both dietary $\mathrm{NAD}^{+}$and $\mathrm{NMN}$ can be transformed into the three forms of the vitamin by a combined action of enzymes of the intestinal mucosa and microbiota (Bogan \& Brenner, 2008).

Numerous lines of evidence indicate that administration of the vitamin NR and its precursor NMN to mice at doses ranging from 100 to $500 \mathrm{mg} / \mathrm{kg} /$ day causes a significant increase in the intracellular content of $\mathrm{NAD}^{+}$in many tissues and organs, which is reflected in improvements in energy metabolism and mitochondrial function. As a result, supplementation of NR or NMN shows both preventive and therapeutic properties in neurodegenerative diseases (e.g., Parkinson's and Alzheimer's), metabolic syndrome (Hartnup's disease), human immunodeficiency virus (HIV), autoimmune diseases, alcohol dependence, anorexia and diseases related to aging that seem to reproduce the symptoms of pellagra (Chi \& Sauve, 2013; Hong, Mo, Zhang, Huang, Wei, \& 2020; Rajman, Chwalek, Sinclair, \& 2018; Ruggieri, Orsomando, Sorci, \& Raffaelli 2015; Yoshino, Baur, \& Imai, 2018). Doses yielding health benefits in mice models are much higher than the amounts of NR and NMN that have been documented to date in a common balanced diet. NMN has been detected in many natural foods, such as broccoli, tomatoes, mushrooms, cabbage, shrimp, avocado and beef meat, with a maximum concentration of $1.88 \mathrm{mg} / 100 \mathrm{~g}$ (Mills et al., 2016), whereas NR has only been documented at micromolar concentrations in milk to date (Trammell, Yu, Redpath, Migaud, \& Brenner, 2016; Ummarino et al., 2017). Considering the beneficial effects attributed to these molecules, it is important to extensively investigate their distribution in food. Beside the natural 
presence of NR in milk, it has been hypothesized that microbial metabolic activities could contribute to its occurrence in fermented food and beverages (Chi \& Sauve, 2013). Indeed, different authors reported the ability of Saccharomyces cerevisiae to actively secrete this vitamin (Bogan et al., 2009; Lu, Kato, \& Lin, 2009). Such evidence prompted us to investigate the presence of NR and its dietary precursors, $\mathrm{NMN}$ and $\mathrm{NAD}^{+}$, in craft beer. Beer is a beverage consumed worldwide that is derived from a biochemical process based on the fermentation of sugary substrates present in the wort beer by the action of yeast (Anderson, Santos, Hildebrand, \& Schug, 2019; Nardini \& Garaguso, 2020). This process is an alcoholic fermentation that leads to the production of ethanol, carbon dioxide and other secondary compounds, such as polyphenols particularly phenolic acids (benzoic and cinnamic acid derivatives) and flavonoids, important for the characterization of the product (Nardini \& Garaguso, 2020). The raw materials necessary for the beer production include water, barley (Hordeum vulgare) and other cereals eventually used, hops (Humulus lupulus), and yeast. Yeast strains used for the brewing process belong to the genus Saccharomyces spp. Traditionally, these yeast strains are classified as yeast for low fermentation, namely, Saccharomyces pastorianus (operating temperature $8-15^{\circ} \mathrm{C}$ ), and yeast for high fermentation, namely, S. cerevisiae (operating temperature $15-23^{\circ} \mathrm{C}$ ). The use of $S$. cerevisiae cultures (top yeast) produces a high fermentation beer (top fermentation) called Ale, in which the yeasts tend to rise to the surface positioning in the foam. In contrast, S. pastorianus (bottom yeast) produces low fermentation (bottom fermentation) in which the yeast at the end of the fermentation process are found on the bottom of the beer based on their ability to flocculate (Lager beer) (Iserentant, 2003; Lodolo, Kock, Axcell, \& Brooks, 2008; Speers, Tung, Durance, \& Stewart, 1992; Verstrepen, Derdelinckx, Verachtert, \& Delvaux, 2003). The brewing process can be divided in four different main phases: malting (transformation of barley into malt), mashing (production of wort), fermentation by yeast (transformation of sugars in ethanol, carbon dioxide and secondary compounds) and downstream processes (maturing, bottling, and packaging) (Anderson et al., 2019). At the end of maturation (generally 3-4 weeks), the beer must be subjected to filtration processes to separate the suspended solids and to pasteurization to produce a 
more stable final product. In a few cases, there is another step of hop addition to the beer. This step,

106 which is called dry hopping, can be performed before fermentation, at the end of fermentation, or 107 during a second fermentation in the bottle. Craft beers, unlike industrial beers, are usually subjected 108 to a second fermentation process in the bottle, by the addition of sugars and yeast. The beer produced 109 in craft breweries differs from industrial beers also because they are consumed unfiltered and 110 unpasteurized (Garofalo et al., 2015). Moreover, craft breweries produce mainly Ale beers, so they

111 utilize predominantly S. cerevisiae strains (Iattici, Catallo, \& Solieri, 2020).

112 In this work, levels of NR and its precursors $\mathrm{NMN}$ and $\mathrm{NAD}^{+}$have been quantified in different craft 113 beers via an enzyme-coupled assay (Ummarino et al., 2017). In addition, laboratory-scale 114 fermentations have been established using different S. cerevisiae strains added to wort or YPD 115 medium to shed light on the mechanism of NR production.

\section{Material and methods}

\subsection{Craft beer and wort sampling}

Ten craft beers of different brewing styles were analyzed for the presence of NR, NMN and NAD ${ }^{+}$. In particular, two samples from different batches of each beer type were collected from two craft breweries located in the Marche region (Central Italy). All the worts used to produce these beers were collected at the end of boiling step and stored at $4^{\circ} \mathrm{C}$. Table 1 summarizes the yeast species and strains, ingredients and alcohol percentage (\%) of the craft beers under study, and Figure 1 shows a flow diagram of their manufacturing process. 
130 The yeast strains S. cerevisiae US-05 (Fermentis Lessafre Italia, Parma, Italy) and S. cerevisiae CBS $1311171^{\mathrm{T}}$ (from the Centraalbureau voor Schimmelcultures, Filamentous fungi and Yeast Collection, 132 The Netherlands) were grown on Yeast Extract Peptone D-glucose (YPD) (yeast extract $10 \mathrm{~g} / \mathrm{L}$, 133 peptone $20 \mathrm{~g} / \mathrm{L}, \mathrm{D}$-glucose $20 \mathrm{~g} / \mathrm{L}$ ) medium at $25^{\circ} \mathrm{C}$ for 72 hours. Yeast strains were inoculated in 134 sterile flasks containing $100 \mathrm{~mL}$ of wort (or $200 \mathrm{~mL}$ YPD) to reach a final concentration of 135 approximately $6 \log _{10} \mathrm{cfu} / \mathrm{mL}$. In a conventional fermentation trial, after 9 days at $21^{\circ} \mathrm{C}$, two different 136 hops were added in pellet form (dry hopping). These hops consisted of amarillo (alpha acid: 9.0\%) $137(4 \mathrm{~g} / \mathrm{L})$ and centennial (alpha acid: 8.5\%) (4 g/L) varieties. After this addition, the maturation 138 continued at $4^{\circ} \mathrm{C}$. At the $16^{\text {th }}$ day of fermentation, dextrose $(7 \mathrm{~g} / \mathrm{L})$ was added; it was dissolved in 600 $139 \mu \mathrm{L}$ of sterile water by heating at $100^{\circ} \mathrm{C}$ for 5 minutes. The brewing process continued at $4{ }^{\circ} \mathrm{C}$ until the $140 \quad 45^{\text {th }}$ day unless otherwise stated.

141 At different days during the fermentation, aliquots of samples were removed to analyze the following: 142 i) the yeast concentration through viable counting on YPD agar (agar $18 \mathrm{~g} / \mathrm{L}$ ) following decimal serial 143 dilutions on sterile peptone water (peptone $1 \mathrm{~g} / \mathrm{L}$ ); ii) the content of $\mathrm{NR}, \mathrm{NMN}$ and $\mathrm{NAD}^{+}$as described 144 in paragraph 2.3.

148 Samples of beers and aliquots from the fermentation trials were subjected to acid-soluble nucleotides 149 extraction. To this end, $0.5 \mathrm{~mL}$ were centrifuged at $16000 \mathrm{x} \mathrm{g}$ for 5 minutes at room temperature 150 before adding $250 \mu \mathrm{L}$ of $1.2 \mathrm{M} \mathrm{HClO}_{4}$. After 15 minutes at $4{ }^{\circ} \mathrm{C}$, samples were centrifuged as 151 described above and $700 \mu \mathrm{L}$ of the supernatants were added to $170 \mu \mathrm{L}$ of $1.0 \mathrm{M} \mathrm{K}_{2} \mathrm{CO}_{3}$ to reach a $\mathrm{pH}$ 152 value of approximately 7.0. Neutralized samples were centrifuged again, and the supernatants were 153 used for the quantitation of NR, NMN and NAD ${ }^{+}$through the enzyme-coupled assay described by 154 Ummarino et al. (2017). Briefly, the coupled assay consists of two consecutive reactions catalyzed 155 by recombinant bacterial NR kinase and recombinant murine NMN adenylyltransferase that 
stoichiometrically convert $\mathrm{NR}$ to $\mathrm{NMN}$ and $\mathrm{NMN}$ to $\mathrm{NAD}^{+}$, respectively. The produced $\mathrm{NAD}^{+}$is then quantified by the fluorometric cycling assay described by Zamporlini et al. (2014).

\subsection{Statistical Analysis}

$\mathrm{NR}, \mathrm{NMN}$ and $\mathrm{NAD}^{+}$data are represented by boxplot that represent the interquartile range (IQR) between the first and third quartiles, and the line inside the plot represents the median (2nd quartile). Data were subjected to one-way ANOVA to examine the development across time. When significant differences were found, Duncan's multiple range test was used. Linear regression model was used to reveal the associations between $\mathrm{NR}, \mathrm{NMN}$ and $\mathrm{NAD}^{+}$as a function of time. A P-value of less than 0.05 was considered statistically significant. All statistical analyses were performed using SPSS 21.0 and R software.

\section{Results and Discussion}

\subsection{NAD ${ }^{+}, N M N, N R$ determination in craft beers}

Ten different types of craft beers were analyzed for the presence of NR, NMN and $\mathrm{NAD}^{+}$as described in paragraph 2.3. All the beers analyzed were prepared with S. cerevisiae, except beer B1, which was produced by $S$. pastorianus. The results of the screening are shown in Figure 2 . To the best of the authors knowledge, this is the first report showing the presence of $\mathrm{NAD}^{+}, \mathrm{NMN}$ and NR in beer. In more detail, $\mathrm{NAD}^{+}$was detected in all the studied beers with the exception of $\mathrm{B} 1$. Its content showed marked variability, even within different batches of the same beer. Levels ranged from $1.10 \mathrm{nmol} / \mathrm{mL}$ to $17.80 \mathrm{nmol} / \mathrm{mL}$ and were intriguingly very similar to those determined in ovine and caprine milk

180 (Ummarino et al., 2017). The highest amount of $\mathrm{NAD}^{+}(\mathrm{P}<0.05)$ was present in the two beers 181 produced using the $S$. cerevisiae strain S-04. In particular the highest value was reached in sample 
B6 followed by sample B5 while lowest amount and minimal differences were observed among the

183 others (Figure 2). These results suggest that S-04 has the capacity among the tested strains to produce and release the highest amount of $\mathrm{NAD}^{+}$during the brewing process. In addition, the only beer lacking $\mathrm{NAD}^{+}$was the one prepared using $S$. pastorianus (sample B1), thus indicating that $\mathrm{NAD}^{+}$production could be species specific. Interestingly, in this latter beer, NR and NMN were also undetectable (Figure 2). Notably, among the beers prepared with S. cerevisiae, all the beers produced with the strain US-05 contained NR. Three of the beers also contained NMN (sample B7, B9 and B10), although at levels generally lower than NR and without differences in the quantity among them (Figure 2, P>0.05). NR concentrations ranged from 0.48 to $3.25 \mathrm{nmol} / \mathrm{mL}$, and these values were unexpectedly very similar to those determined in bovine milk (from 0.5 to $3.6 \mathrm{nmol} / \mathrm{mL}$ ) (Trammell et al., 2016; Ummarino et al., 2017). In particular, NR was mostly detected in samples B9 and B10.

193 However, no difference in concentration between the two was observed (Figure 2, P>0.05). 194 Furthermore, samples B7 and B8 showed comparable quantities of NR but always significantly lower than B9 and B10 (Figure 2, P<0.05). In contrast to NR, NMN was not always present in both tested 196 batches of beer, and when present, its content was approximately $0.9 \mathrm{nmol} / \mathrm{mL}$. This content closely 197 resembles that measured in ovine and donkey milk, where NMN ranges from 0 to $1.0 \mathrm{nmol} / \mathrm{mL}$ 198 (Ummarino et al., 2017). In general, NMN levels in beer and milk are lower than those measured in 199 other foods, such as tomato, avocado and beef meat (from $0.78 \mathrm{nmol} / \mathrm{mg}$ to $4.79 \mathrm{nmol} / \mathrm{mg}$ ) (Mills et 200 al., 2016).

201 All the worts were negative for the presence of NR and its metabolic precursors (data not shown). 202 The data obtained in the present study clearly indicate that the production of the three metabolites is 203 a typical feature of the S. cerevisiae species and is strain dependent. 
207 Screening of NR, NMN and $\mathrm{NAD}^{+}$in the different craft beers indicated that only the beers produced 208 with S. cerevisiae strain US-05 contained all the three vitamers (Figure 2). Prompted by these results, 209 a replicate of the beer B9 on a laboratory scale (B9L) was established to monitor the production of 210 the molecules during the entire fermentation process. The B9 sample was chosen based on the 211 availability of the corresponding wort by the brewery. The fermentation was performed as described 212 in paragraph 2.2, and the production of the three metabolites as well as the viable yeast counts were 213 monitored during the entire process (Figure 3A).

214 On the $2^{\text {nd }}$ day of fermentation, the concentration of the yeast strain significantly increased from the 215 starting inoculum $(\mathrm{P}<0.05)$ reached the value of $7.5 \log _{10} \mathrm{cfu} / \mathrm{mL}$ and then remained constant until 216 the end of the process $(\mathrm{P}<0.05)$. Different fluctuations in the levels of the metabolites were recorded. 217 After an initial lag of approximately 2 days, the amount of NR significantly increased, exhibiting a 218 burst after the addition of the hop, i.e., after the $9^{\text {th }}$ day. In fact, the value of NR shifted from 1.3 $219 \mathrm{nmol} / \mathrm{mL}$ on the $9^{\text {th }}$ day to $3.4 \mathrm{nmol} / \mathrm{mL}$ on the $14^{\text {th }}$ day, representing an approximately 3 -fold 220 increase. The linear regression model showed a significant increase in the production of NR across 221 time (Adjusted R-squared: 0.9694, p-value: $<0.05)$. The addition of sugar after $16^{\text {th }}$ days did not 222 affect the trend of NR. The trend for $\mathrm{NAD}^{+}$was very different from that of NR. In more detail, in the 223 first two days of the process, this metabolite significantly increased to approximately $3 \mathrm{nmol} / \mathrm{mL}$ $224(\mathrm{P}<0.05)$ and remained at this level until the addition of the hops. After the $9^{\text {th }}$ day, it sharply 225 decreased to very low levels (Adjusted R-squared: 0.2701, p-value< 0.05). Regarding NMN, after a 226 sharp increase in the first two days to a value similar to that of $\mathrm{NAD}^{+}$, it remained constant until the $22723^{\text {rd }}$ day and then started to slowly decrease (Adjusted R-squared: 0.003437, p-value: 0.2992).

228 A comparison of the levels of the molecules of interest in the B9 beer (Figure 2) and in the lab-scale 229 beer B9L on the last day of the fermentation (Figure 3A) revealed that the final amount of NR in B9L 
was considerably increased compared with that in $\mathrm{B} 9$ beer, whereas $\mathrm{NAD}^{+}$was lower. On the other

231 hand, NMN levels were very similar.

232 The differences in NR and $\mathrm{NAD}^{+}$content between $\mathrm{B} 9$ and $\mathrm{B} 9 \mathrm{~L}$ samples might be due to several 233 reasons. In fact, although we used the same ingredients at the same concentrations as in the brewery, 234 the yeast strain used by the brewery was in a lyophilized form (not grown on YPD for 72 hours), and 235 the second fermentation occurred in a closed bottle in the brewery. Furthermore, the time elapsed 236 from bottling to sampling was unknown.

237 The results in Figure 3A suggest that the addition of the hops might be responsible for boosting NR and decreasing $\mathrm{NAD}^{+}$during the fermentation trial. This behavior was confirmed by the linear regression model where a positive relationship among $\mathrm{NAD}^{+}$and NR was observed (Adjusted Rsquared: 0.3632 , p-value $<0.05)$.

241 To better define the role of hops in the change in metabolites levels, a control fermentation trial without the addition of hops was established (Figure 3B). After the initial increase to approximately $3 \mathrm{nmol} / \mathrm{mL}, \mathrm{NAD}^{+}$continued to slightly increase throughout the fermentation process (Adjusted Rsquared: 0.6937, p-value < 0.05), whereas NMN remained constant (Adjusted R-squared: 0.1112, pvalue $<0.05)$. NR showed a very slight increase from the $8^{\text {th }}$ day until the end of the fermentation 246 (Adjusted R-squared: 0.7835, p-value< 0.05). Altogether, these results suggest that the addition of hops stimulates NR production and induces degradation of both $\mathrm{NAD}^{+}$and NMN. However, the relationship was verified by the linear model only by considering the behavior of NR and NAD (Adjusted R-squared: 0.56, p-value< 0.05).

S. cerevisiae cells constitutively produce, release and import NR (Bogan et al., 2009; Lu et al., 2009), 251 whereas no information is available on the ability of plant cells to release NR. It is therefore tempting to hypothesize that hops might enhance the yeast's ability to produce and release the vitamin. In this view, yeast cells would facilitate $\mathrm{NAD}^{+}$supply to hop cells by providing the NR precursor. Metabolic interaction between different cell-types through the exchange of extracellular metabolites is a wellknown mechanism, and evidence has been provided that different cell types might support each 
other's $\mathrm{NAD}^{+}$pools by providing $\mathrm{NR}$ as $\mathrm{NAD}^{+}$precursor (Kulikova et al., 2015). Morover, it is interesting to note that Steyer, Tristam, Clayeux, Heitz, \& Laugel (2017) highlighted a synergy between several yeast strains and hop varieties on beer volatile compounds production, thus indicating that an interaction between hop compounds and yeast metabolism exists although it remains to be investigated. Unfortunately, the lack of information on the regulation of intracellular 261 NR generation and release does not allow to explain the mechanism underlying the metabolic interaction between yeast and hop cells. Furthermore, to the authors' knowledge, data regarding NR, $\mathrm{NMN}$ and $\mathrm{NAD}^{+}$dynamics during a craft beer production is lacking in the scientific literature, thereby preventing further comparison.

\subsection{Effect of wort and S. cerevisiae strain on the production of $N R, N M N$ and $N A D^{+}$}

The present study also investigated whether the presence of the wort was required for the NR bursting effect exerted by the hops. To this end, a fermentation trial substituting the wort with YPD medium and determining the concentrations of the molecules of interest on different days was performed. As shown in Figure 4A, the change in metabolites levels during the process closely resembled that observed in the fermentation of wort. Indeed, in YPD, the addition of hops caused a rapid increase in

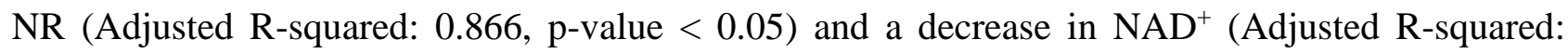
0.1699 , p-value $<0.05$ ) thus indicating that the presence of wort is not essential for the production of the vitamin. Even in YPD a linear trend was observed by the consumption of $\mathrm{NAD}^{+}$and the production of NR (Adjusted R-squared: 0.2964, p-value < 0.05). Dextrose was not added in these fermentation trials since it was previously demonstrated that it did not influence the vitamer trends. It was therefore asked whether the presence of the yeast was required for the effect of the hops and whether the effect was strain specific. Figure $4 \mathrm{~B}$ shows that NR and NAD ${ }^{+}$are not produced in the absence of yeast. On the other hand, a slight amount of NMN was produced when hops were added 
in the presence of the S. cerevisiae strain US-05 and decreased to undetectable levels at the end of 283 the process.

284 Figure 4C shows the levels of the metabolites during the fermentation of YPD inoculated with $S$. 285 cerevisiae strain CBS $1171^{\mathrm{T}}$. The tendency of NR to increase following the addition of hops was 286 observed also in the presence of this strain. Strain CBS $1171^{\mathrm{T}}$ released NR on the $4^{\text {th }}$ day, whereas 287 the vitamin was detectable on the $9^{\text {th }}$ day with the strain US-05 (Adjusted R-squared: 0.9138, p-value $288<0.05)$. Moreover, in the absence of hops, NR decreased in the strain US-05 under sustained 289 fermentation, whereas it continued to slowly increase when strain CBS $1171^{\mathrm{T}}$ was present (Adjusted 290 R-squared: 0.6907, p-value < 0.05). NMN production was different in the two fermentations. The 291 strain US-05 released NMN from the beginning of the process, and the addition of hops did not change 292 its concentration up to the $15^{\text {th }}$ day. Then, a progressive decrease was noted (Adjusted R-squared: 293 0.04175, p-value: 0.2346). On the other hand, strain CBS $1171^{\mathrm{T}}$ produced NMN only after the 294 addition of hops, and the levels slowly decreased during the fermentation (Adjusted R-squared: 295 0.3007, $\mathrm{p}$-value < 0.05). The trend of $\mathrm{NAD}^{+}$production was similar in the two fermentations. In fact, 296 the addition of hops also caused a decrease in NAD ${ }^{+}$levels with the CBS $1171^{\mathrm{T}}$ strain. CBS $1171^{\mathrm{T}}$ 297 released more $\mathrm{NAD}^{+}$than US-05.

298 Altogether, these results indicated that both hops and yeast are required during the brewing process 299 to increase NR production. Moreover, the NR bursting effect seems to be $S$. cerevisiae strain and wort 300 independent. Further studies to elucidate the interaction between hops and S. cerevisiae cells at 301 intracellular metabolic level could shed more light on the NR bursting effect exerted by hops.

\section{Conclusion}

In the present work, for the first time, the presence of NR and its dietary precursors NMN and NAD ${ }^{+}$ in craft beers was quantitatively assessed, suggesting potential beneficial properties of such a low alcoholic beverage. The presence of the three vitamers in the beers under study was $S$. cerevisiae 
strain-dependent. Overall, all craft beers prepared with different S. cerevisiae strains contained

$309 \mathrm{NAD}^{+}$, further highlighting the potentialities of microorganisms to act as biological factories for

310 beneficial molecules to humans. By reproducing a lab-scale fermentation process either in wort and

311 YPD medium, a significant increase in NR levels was observed after the addition of hops, and both

312 the yeast S. cerevisiae and hops are required for such a burst to occur, thus indicating that a yeast and

313 hops sinergy on NR production occurs. The present study represents the first attempt to provide an

314 experimental model to study the hop-yeast interaction at metabolic and molecular levels. Finally,

315 these findings open new intriguing perspectives for the development of innovative fermented foods 316 naturally enriched in NR and its precursors.

\section{Declaration of Competing Interest}

The authors declare that they have no known competing financial interests or personal relationships that could have appeared to influence the work reported in this paper.

\section{Acknowledgements}

Authors would like to thank the breweries "Birrificio SanGermano18" and "Birrificio del Gomito" and their owners (Corrado Pirani and Paolo Ragni, respectively) for their voluntary help in the supply of yeast strains, beer and wort samples and information about brewing production. This work was partly supported by PRIN2017CBNCYT to NR. 
Anderson, H. E., Santos, I. C., Hildenbrand, Z. L., \& Schug, K.A. (2019). A review of the analytical methods used for beer ingredient and finished product analysis and quality control. Analytica Chimica Acta, 1085, 1-20.

Bieganowski, P., \& Brenner, C. (2004). Discoveries of nicotinamide riboside as a nutrient and conserved NRK genes establish a Preiss-Handler independent route to NAD+ in fungi and humans. Cell, 117, 495-502.

Bogan, K. L., \& Brenner, C. (2008). Nicotinic acid, nicotinamide, and nicotinamide riboside: a molecular evaluation of NAD+ precursor vitamins in human nutrition. Annual Review of Nutrition, 28, 115-130.

Bogan, K. L., Evans, C., Belenky, P., Song, P., Burant, C. F., Kennedy, R., \& Brenner, C. (2009). Identification of Isn1 and Sdt1 as glucose-and vitamin-regulated nicotinamide mononucleotide and nicotinic acid mononucleotide 5'-nucleotidases responsible for production of nicotinamide riboside and nicotinic acid riboside. Jounal of Biological Chemistry, 284, 34861-34869.

Chi, Y., \& Sauve, A. A. (2013). Nicotinamide riboside, a trace nutrient in foods, is a vitamin B3 with effects on energy metabolism and neuroprotection. Current Opinion in Clinical Nutrition \& Metabolic Care, 16, 657-661.

Garofalo, C., Osimani, A., Milanović, V., Taccari, M., Aquilanti, L., \& Clementi, F. (2015). The Occurrence of Beer Spoilage Lactic Acid Bacteria in Craft Beer Production. Journal of Food Science, 80(12), M2845-M2852. doi: 10.1111/1750-3841.13112

Hong, W., Mo, F., Zhang, Z., Huang, M., \& Wei, X. (2020). Nicotinamide Mononucleotide: A Promising Molecule for Therapy of Diverse Diseases by Targeting NAD+ Metabolism. Frontiers in Cell and Developmental Biology, https://doi.org/10.3389/fcell.2020.00246.

Iattici, F., Catallo, M., \& Solieri, L. (2020). Designing New Yeasts for Craft Brewing: When Natural Biodiversity Meets Biotechnology. Beverages, 6(3), https://doi:10.3390/beverages6010003.

Iserentant, D. (2003). Beers: recent technological innovations in brewing. In: Lea, A.G.H., Piggott, J.R., (Second ed.), Fermented Beverage Production, Springer, Boston, pp. 41-58. 
Kulikova, V., Shabalin, K., Nerinovski, K., Dölle, C., Niere, M., Redpath,...Nikiforov, A. (2015). Generation, Release, and Uptake of the NAD Precursor Nicotinic Acid Riboside by Human Cells. Journal of Biological Chemistry, 290(45), 27124-37. doi: 10.1074/jbc.M115.664458.

Lodolo, E. J., Kock, J. L. F., Axcell, B. C., \& Brooks, M. (2008). The yeast Saccharomyces cerevisiae-the main character in beer brewing. Fems Yeast Research, 8, 1018-1036.

Lu, S.-P., Kato, M., \& Lin, S.-J. (2009). Assimilation of Endogenous Nicotinamide Riboside Is

Essential for Calorie Restriction-mediated Life Span Extension in Saccharomyces cerevisiae. Jounal of Biological Chemistry, 284, 17110-17119.

Mills, K. F., Yoshida, S., Stein, L. R., Grozio, A., Kubota, S., Sasaki, Y.,...Imai, S. I. (2016). Longterm administration of nicotinamide mononucleotide mitigates age-associated physiological decline in mice. Cell Metabolism, 24, 795-806.

Nardini, M., \& Garaguso, I. (2020). Characterization of bioactive compounds and antioxidant activity of fruit beers. Food Chemistry, 305, 125437.

Rajman, L., Chwalek, K., \& Sinclair, D. A. (2018). Therapeutic potential of NAD-boosting molecules: the in vivo evidence. Cell Metabolism, 27, 529-547.

Ruggieri, S., Orsomando, G., Sorci, L., \& Raffaelli, N. (2015). Regulation of NAD biosynthetic enzymes modulates NAD-sensing processes to shape mammalian cell physiology under varying biological cues. Biochimica et Biophysica Acta, 1854, 1138-1149.

Speers, R. A., Tung, M. A., Durance, T. D., \& Stewart, G. G. (1992). Biochemical aspects of yeast flocculation and its measurement: a review. Journal of the Institute of Brewing, 98, 293-300.

Steyer, D., Tristram, P., Clayeux, C., Heitz, F., \& Laugel, B. (2017). Yeast strains and hop varieties synergy on beer volatile compounds. Brewing Science, 70, 131-141.

Trammell, S. A. J., Yu, L., Redpath, P., Migaud, M. E, \& Brenner, C. (2016). Nicotinamide Riboside Is a Major NAD+ Precursor Vitamin in Cow Milk. The Journal of Nutrition, 146, 957-63.

Ummarino, S., Mozzon, M., Zamporlini, F., Amici, A., Mazzola, F., Orsomando, G.,... \& Raffaelli, N. (2017). Simultaneous quantitation of nicotinamide riboside, nicotinamide mononucleotide 
and nicotinamide adenine dinucleotide in milk by a novel enzyme-coupled assay. Food Chemistry, 221, 161-168.

386 Verstrepen, K. J., Derdelinckx, G., Verachtert, H., \& Delvaux, F. R. (2003). Yeast flocculation: what brewers should know. Applied Microbiology and Biotechnology, 61, 197-205.

388 Yoshino, J., Baur, J. A., \& Imai, S. I. (2018). NAD+ intermediates: the biology and therapeutic potential of NMN and NR. Cell Metabolism, 273, 513-528.

390 Zamporlini, F., Ruggieri, S., Mazzola, F., Amici, A., Orsomando, G., \& Raffaelli, N. (2014). Novel assay for simultaneous measurement of pyridine mononucleotides synthesizing activities 281, 5104-5119. 
412 Figure 1. Flow diagram of manufacture of the craft beers B1-B8 (A); Flow diagram of manufacture 413 of the craft beers B9 and B10 (B).

415 Figure 2. Boxplots showing the levels NR, NMN, NAD ${ }^{+}$concentrations $(\mathrm{nmol} / \mathrm{mL})$ detected in the 416 different craft beers under study.

Different letters at the base of the boxes indicate significant differences for each metabolite among 419 beers $(\mathrm{P}<0.05)$

Figure 3. NR, NMN and $\mathrm{NAD}^{+}$concentrations in $\mathrm{B} 9 \mathrm{~L}(\mathrm{~A})$ and control (B) during the fermentation process.

424 Measurements were performed in duplicate and the means \pm standard deviation were reported.

Figure 4. Effect of hop addition on NR, NMN and $\mathrm{NAD}^{+}$levels in YPD medium inoculated with $S$. cerevisiae strain US-05 (A), without yeast inoculation (B), and inoculated with S. cerevisiae strain CBS $1171^{\mathrm{T}}(\mathrm{C})$.

430 Hop was added at the $9^{\text {th }}$ day. Measurements were performed in duplicate and the means \pm standard 431 deviation were reported. 
Table 1. Saccharomyces yeast strains, ingredients and alcohol percentage (\%) in craft beer samples

\begin{tabular}{lllll}
\hline Sample & Yeast species & Strain & Ingredients & \% alcohol \\
\hline B1 & Saccharomyces pastorianus & $\mathrm{W} 34 / 70$ & $\mathrm{H}_{2} \mathrm{O}$, hop, yeast, sugar, barley and wheat malt & 6.0 \\
B2 & Saccharomyces cerevisiae & $\mathrm{S}-33$ & $\mathrm{H}_{2} \mathrm{O}$, hop, yeast, sugar, barley malt & 5.5 \\
B3 & Saccharomyces cerevisiae & $\mathrm{WB}-06$ & $\mathrm{H}_{2} \mathrm{O}$, hop, yeast, sugar, barley and wheat malt, wheat & 6.3 \\
B4 & Saccharomyces cerevisiae & $\mathrm{WB}-06$ & $\mathrm{H}_{2} \mathrm{O}$, hop, yeast, sugar, barley and wheat malt & 5.8 \\
B5 & Saccharomyces cerevisiae & $\mathrm{S}-04$ & $\mathrm{H}_{2} \mathrm{O}$, hop, yeast, sugar, barley malt & 6.3 \\
B6 & Saccharomyces cerevisiae & $\mathrm{S}-04$ & $\mathrm{H}_{2} \mathrm{O}$, hop, yeast, sugar, barley and wheat malt & 6.6 \\
B7 & Saccharomyces cerevisiae & $\mathrm{US}-05$ & $\mathrm{H}_{2} \mathrm{O}$, hop, yeast, sugar, barley and wheat malt & 6.6 \\
B8 & Saccharomyces cerevisiae & US-05 & $\mathrm{H}_{2} \mathrm{O}$, hop, yeast, sugar, barley malt & 5.4 \\
B9 & Saccharomyces cerevisiae & US-05 & $\mathrm{H}_{2} \mathrm{O}$, hop, yeast, sugar, barley malt, oat flakes & 5.5 \\
B10 & Saccharomyces cerevisiae & US-05 & $\mathrm{H}_{2} \mathrm{O}$, hop, yeast, sugar, barley and wheat malt, oat flakes & 5.5 \\
\hline
\end{tabular}




\section{Fig. 1}

A)

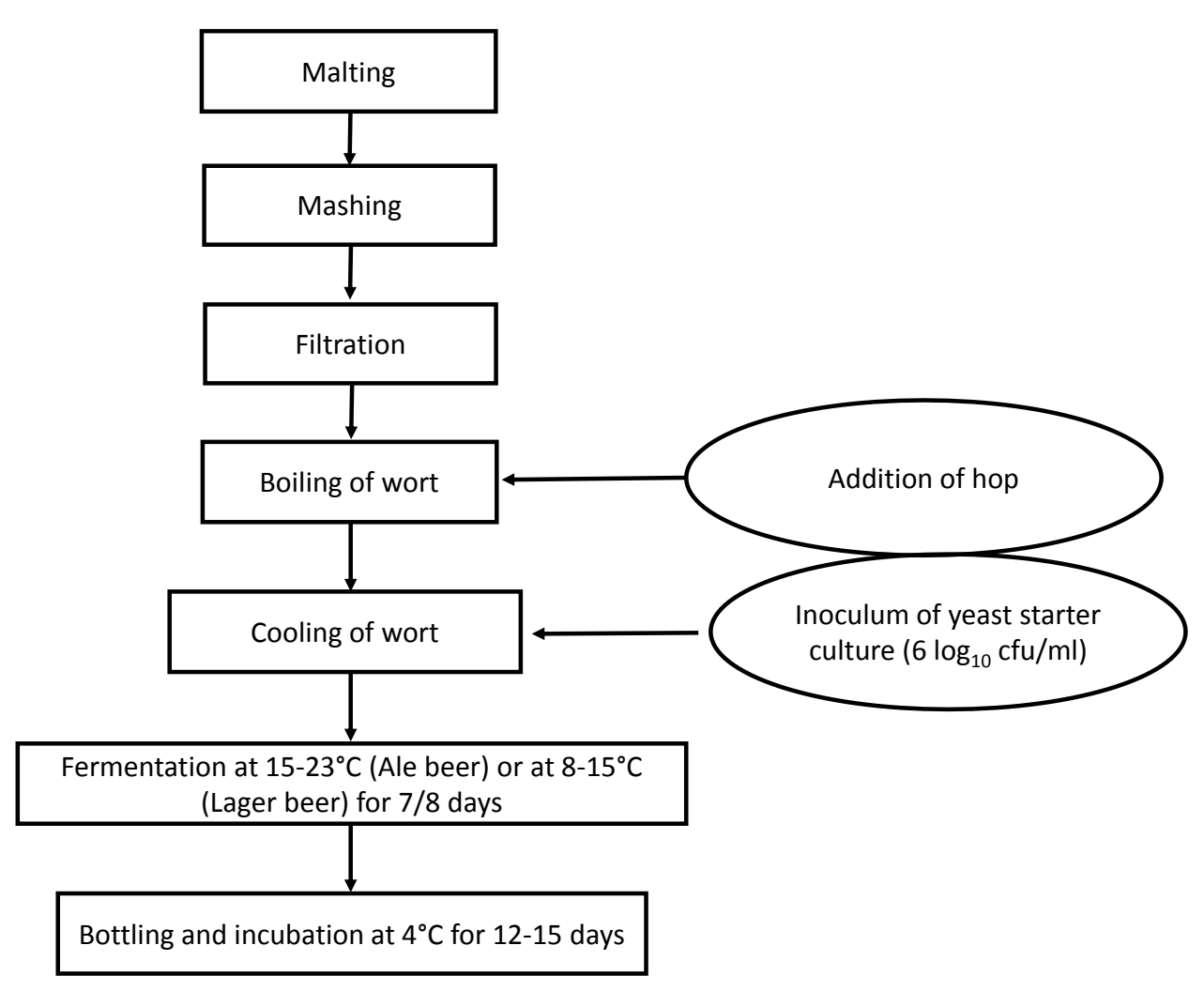

B)

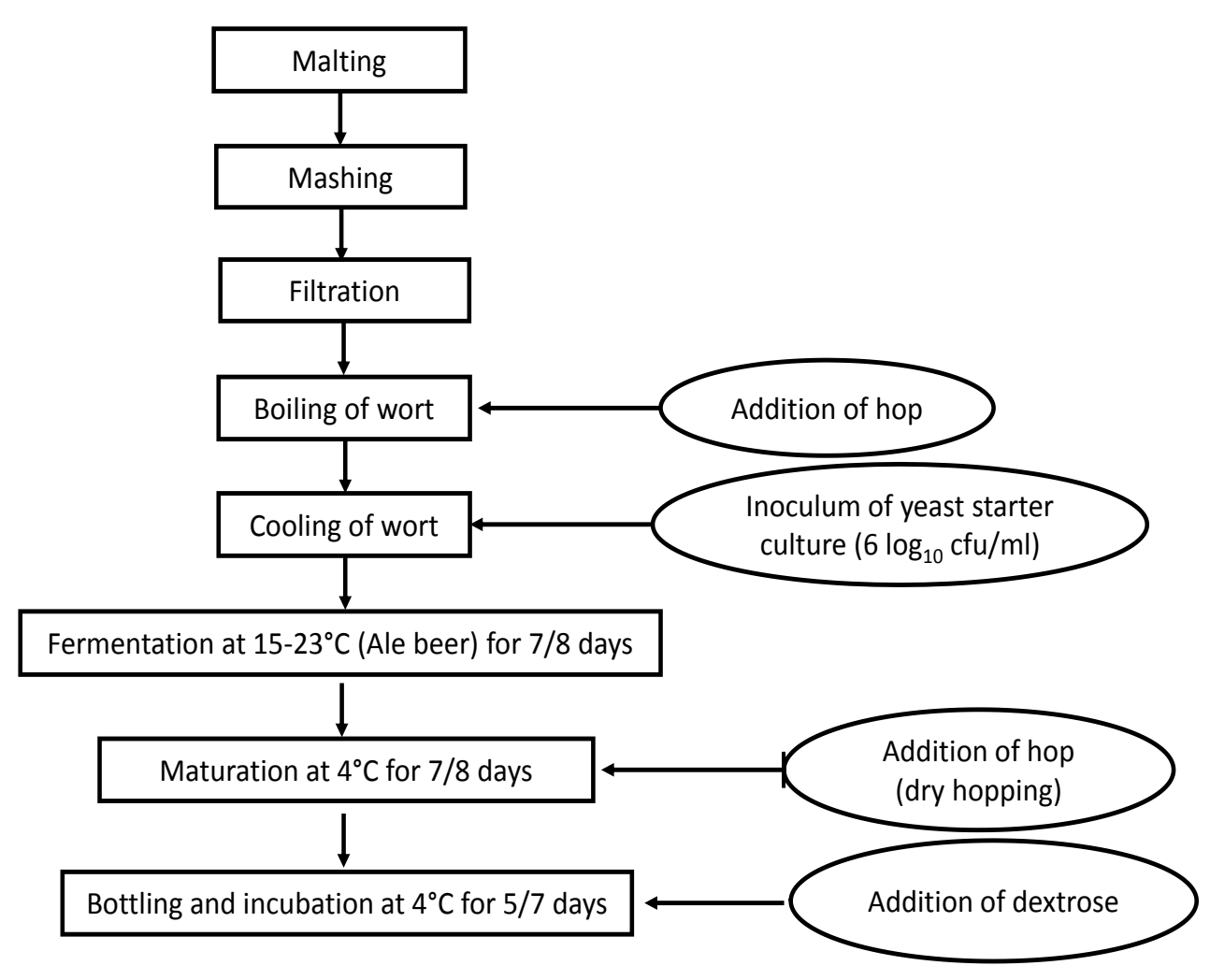


Fig. 2.

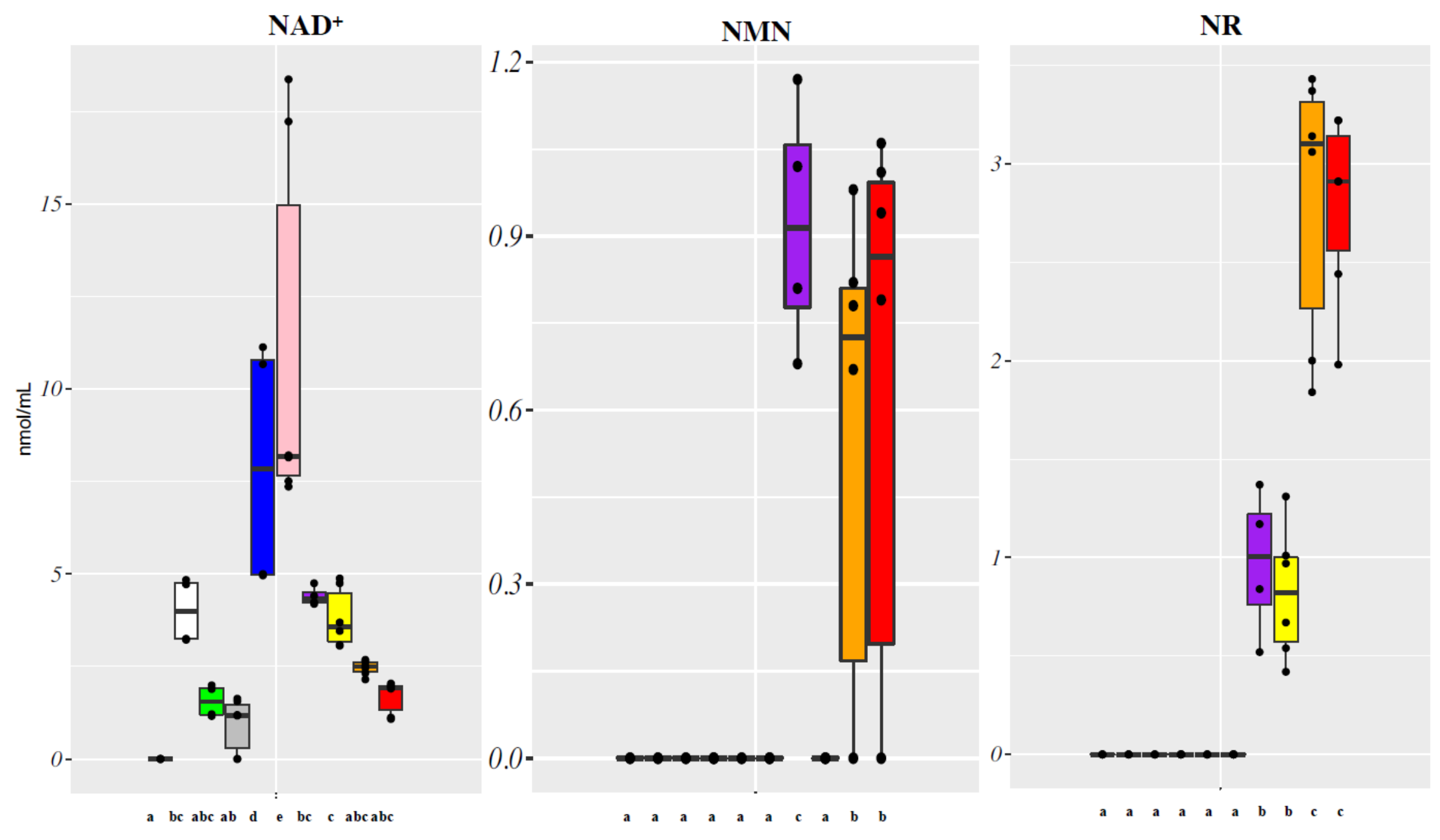

\section{Legend}

B1 S. pastorianus W34/70

官 B2 S. cerevisiae S-33

官 B3 S. cerevisiae WB-06

白 B4 S. cerevisiae WB-06

B5 S. cerevisiae S-04

官 $\mathrm{B} 6$ S. cerevisiae $\mathrm{S}-04$

B7 S. cerevisiae US-05

官 B8 S. cerevisiae US-05

由 B9 S. cerevisiae US-05

B10S. cerevisiae US-05 
Fig. 3

A)

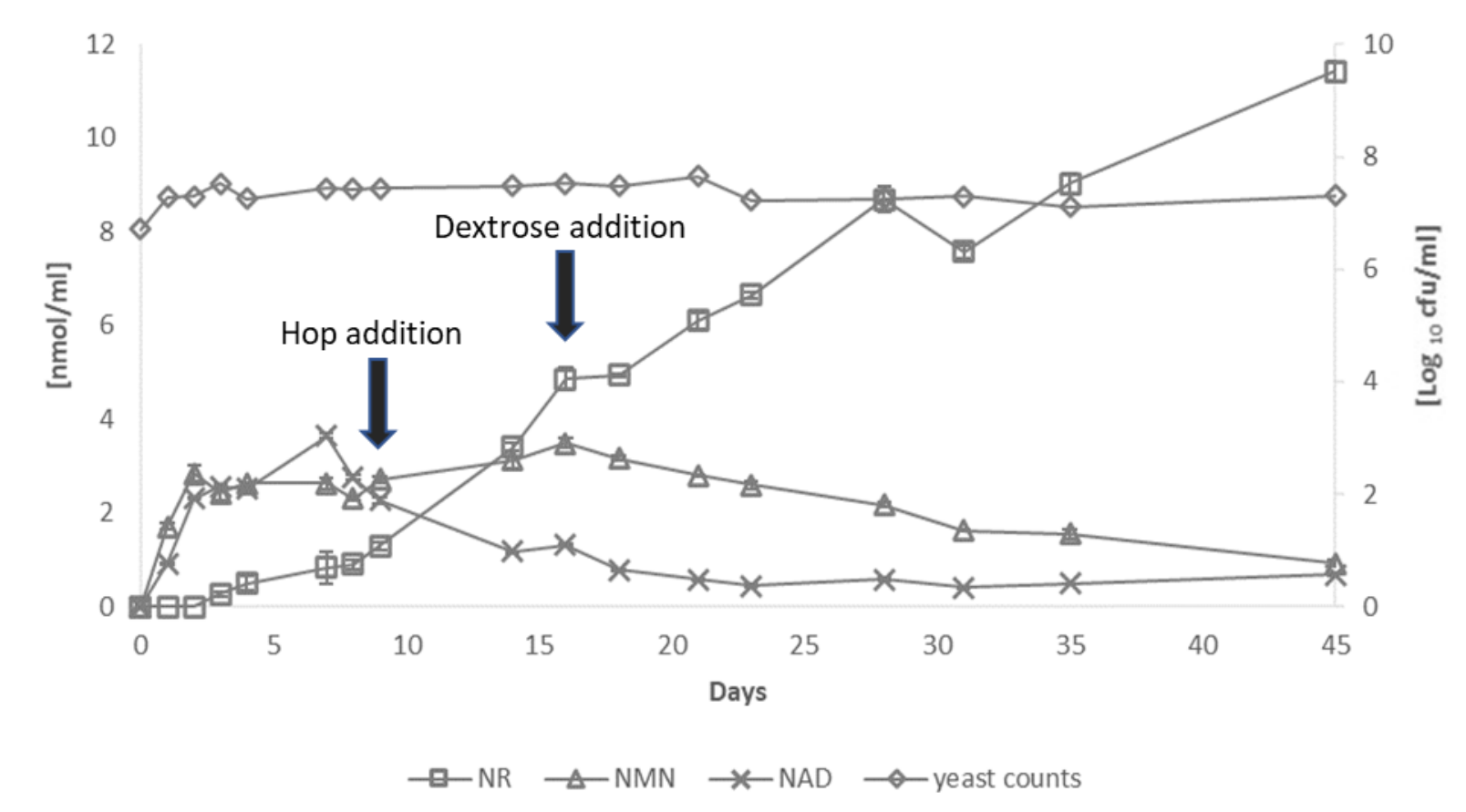

B)

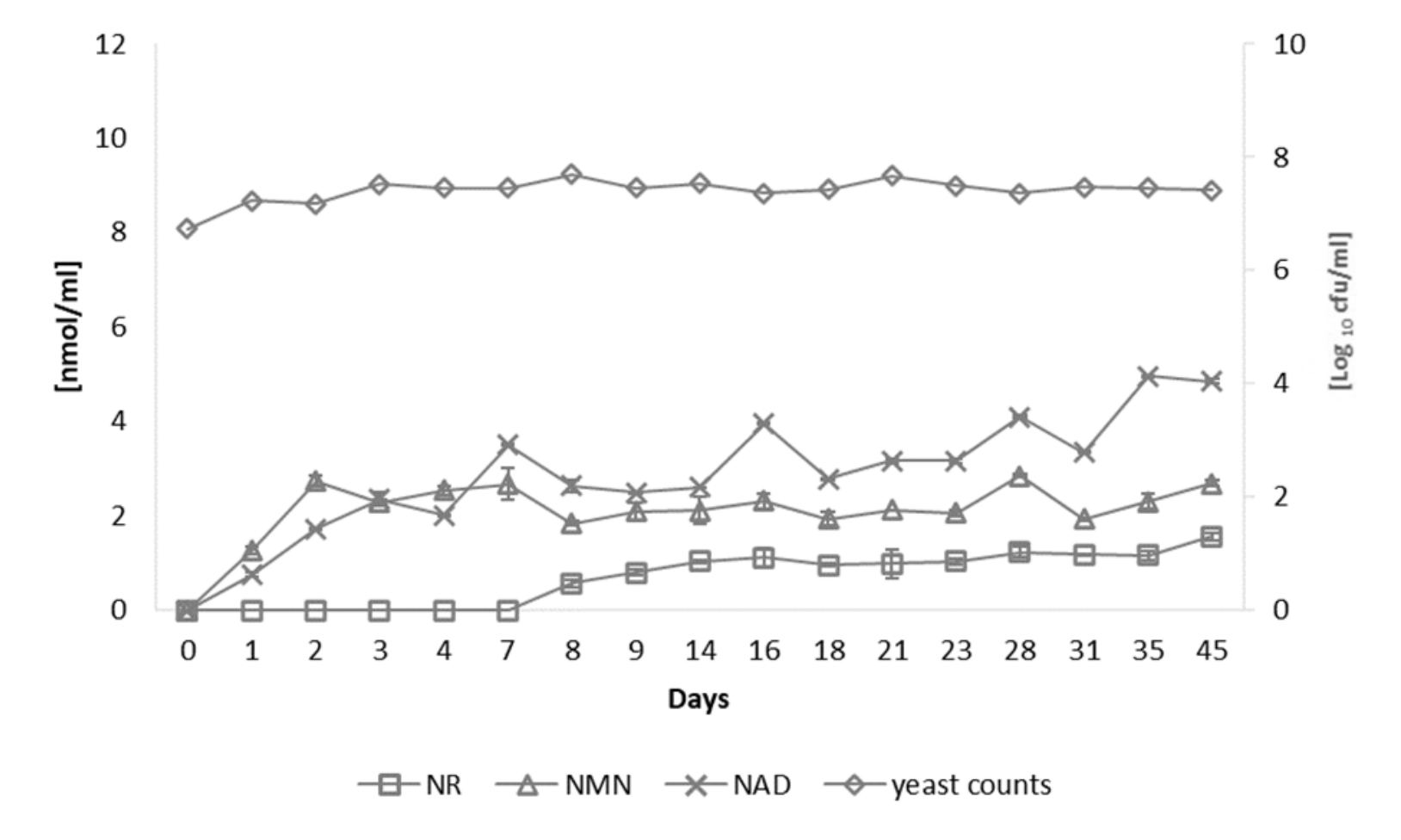


Fig. 4

A)
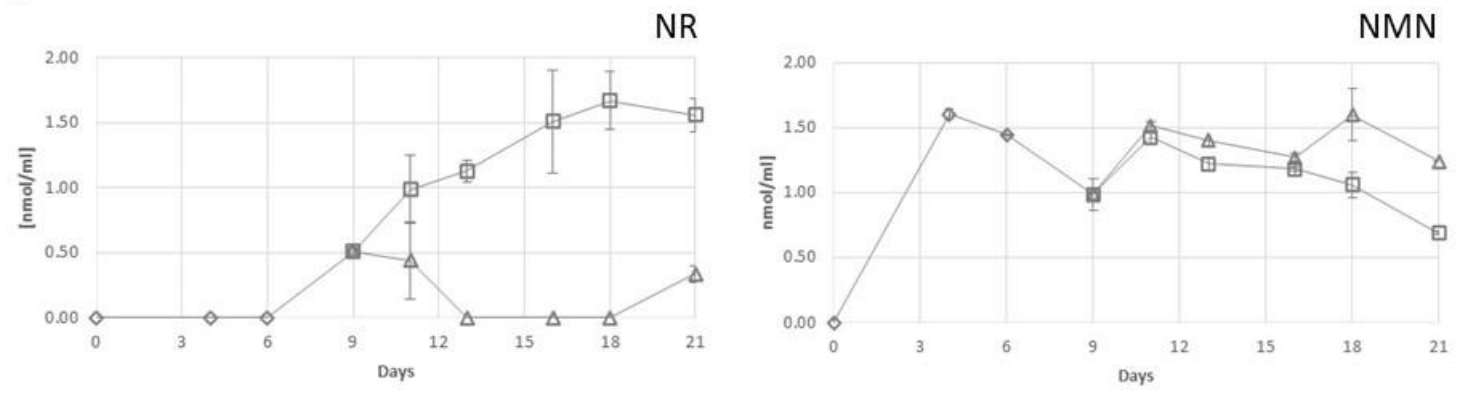

- NMN $\square-$ NMN+HOP $\triangle-N M N-H O P$

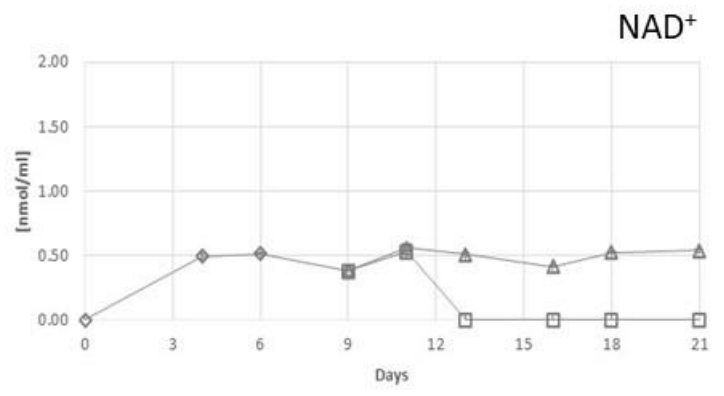

B)
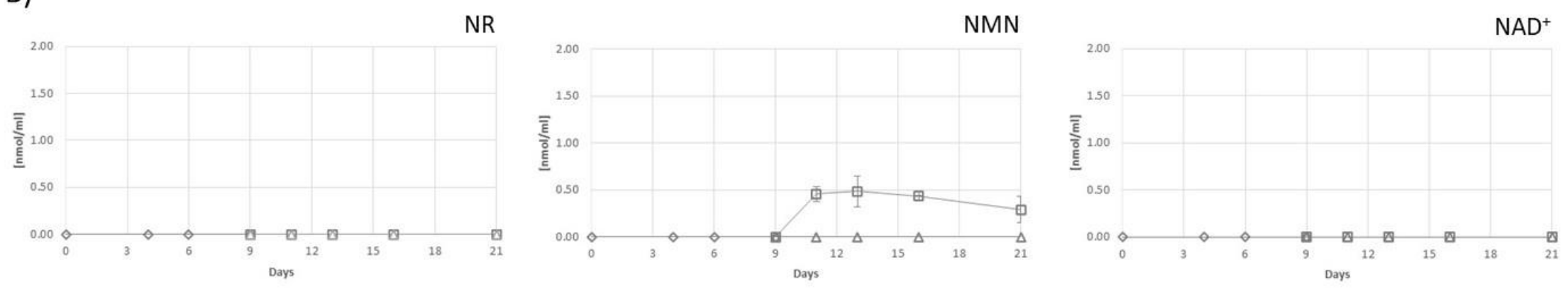

C)

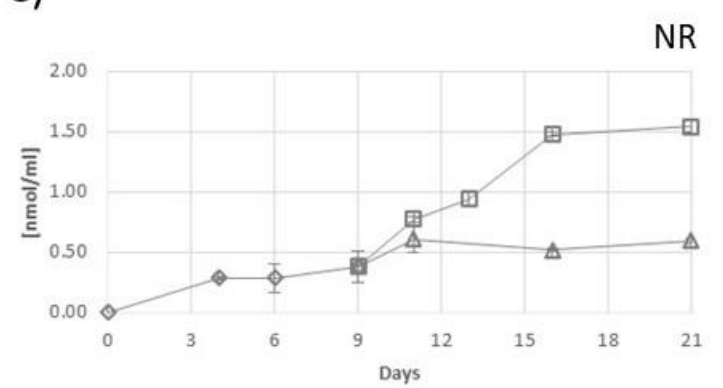

$\rightarrow N R \rightarrow-N R+H O P \rightarrow-N R \cdot H O P$
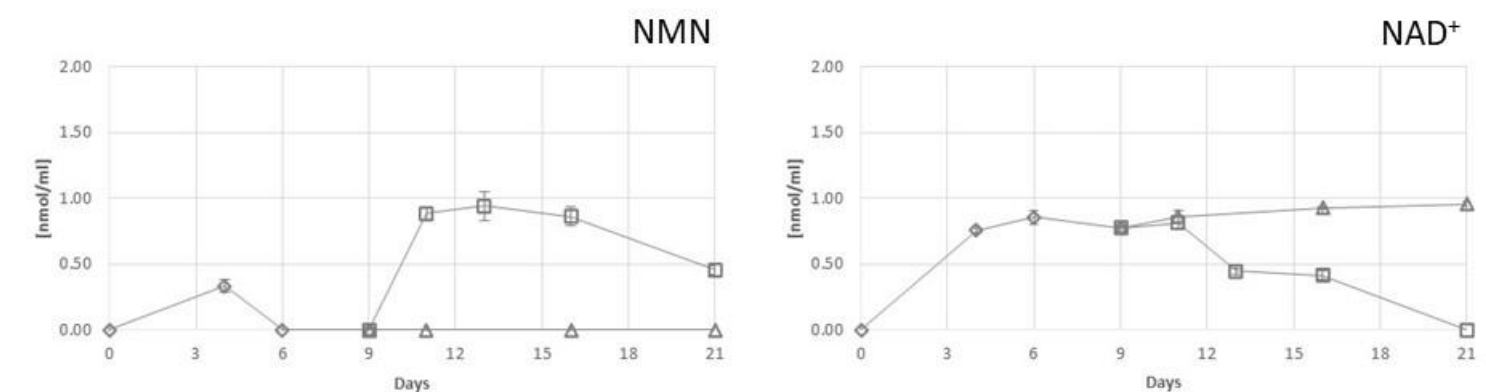

$\rightarrow$ NMN $\square-N M N+$ HOP $\triangle-N M N-H O P$

$\rightarrow$ NAD - Q-NAD+HOP $\triangle$ NAD. HOP 